\title{
Are IgG antibodies to heat shock proteins HSP27 and HSP60 useful markers in endometrial cancer and cervical cancer?
}

\author{
Piotr Bodzek ${ }^{1} \mathbb{D}$, Bogdan Szymala $\left.{ }^{1} \mathbb{(}\right)$, Aleksandra Damasiewicz-Bodzek ${ }^{2} \mathbb{(}$, Iwona Janosz ${ }^{1} \mathbb{D}$, \\ Lukasz Witek ${ }^{1}{ }^{\oplus}$, Anita Olejek ${ }^{1}$ \\ ${ }^{1}$ Department of Gynaecology, Obstetrics and Oncological Gynaecology, Faculty of Medical Sciences in Zabrze, \\ Medical University of Silesia, Bytom, Poland \\ ${ }^{2}$ Department of Chemistry, Faculty of Medicine and Division of Dentistry, Medical University of Silesia, Zabrze, Poland
}

\begin{abstract}
Objectives: Heat shock proteins are overexpressed in many human malignancies. The role of heat shock proteins as a therapeutic target in cancer as well as their association with drug resistance were widely documented. The aim of this study was to evaluate the concentration of IgG class HSP27 and HSP60 antibodies in serum of patients with endometrial and cervical cancer, as well as to analyse the variability of concentrations of the examined antibodies depending on the cancer stage. Material and methods: The study included 59 women with adenocarcinoma of the endometrium and 36 women with cervical cancer, the control group consisted of 54 healthy women. The concentrations of IgG class antibodies against the tested heat shock proteins were determined by an immunoenzymatic assay (ELISA) using commercial assays.

Results: In both endometrial and cervical cancer, the serum concentration of IgG anti-HSP27 antibody was significantly higher than in the healthy control group. The concentration of IgG anti-HSP60 antibody in endometrial cancer, cervical cancer and healthy control was similar.

The median lgG anti-HSP27 antibody serum concentration of endometrial cancer patients was not correlated with FIGO-stage. In cervical cancer inverse correlation between concentration of this antibody and FIGO stage was observed.

The median IgG anti-HSP60 antibody concentration in serum of endometrial cancer patients was lower in FIGO stage I and II compared to FIGO stage IV and in FIGO stage IA compared to FIGO stage IB. Concentrations of examined antibodies correlated positively with each other, both in the group of women with cancer and in the group of healthy women. The strongest correlations were found in the group of patients with endometrial cancer.

Conclusions: Concentration of anti-HSP27 antibody could help in detection of cervical and endometrial cancer. We need to look for the cut-off point in large cohort studies. Anti-HSP27 and anti-HSP60 antibodies should be further evaluated for their potential usage as biomarkers in cervical and endometrial cancer as they shown some correlation with stage of disease. Key words: endometrial cancer; cervical cancer; heat shock proteins
\end{abstract}

Ginekologia Polska 2021; 92, 11: 760-766

\section{INTRODUCTION}

Endometrial cancer is the most common malignancy of the female reproductive organs, and approximately one-fifth of patients are diagnosed at an advanced stage [1]. The incidence of this cancer is steadily increasing due to rising standards of living in the populations of developed countries [2,3]. Although this neoplasm is most often diagnosed at the first clinical stage according to FIGO [4], there is still a search for simple tumour markers that allow the diagnosis and identification of women from high-risk groups. The reason being the imperfection of the applied diagnostic methods, mainly an endometrial biopsy, which, due to technical errors during the procedure or an insufficient amount of material, have a high percentage of false negative results [5].

\section{Corresponding author:}

Piotr Bodzek

Department of Gynaecology, Obstetrics and Oncological Gynaecology, Faculty of Medical Sciences in Zabrze Medical University of Silesia, 15 Batorego St, 41-902 Bytom, Poland e-mail: piotr.bodzek@sum.edu.pl 
Cervical cancer is the second most common cancer in women [6]. Its occurrence is steadily increasing, especially in developing countries [7]. Lack of awareness and insufficient care for women's health are possible reasons for the rising incidence rates in recent years [8]. Additionally, the pathogenesis of this cancer is unclear and may be due to recurrent genetic changes, microenvironments, lifestyle, smoking, etc. [9]. Various studies have shown that persistent HPV infection is a major causative factor in cervical cancer, but only a small proportion of patients with HPV infection will eventually progress to cervical cancer. In cervical cancer patients, the mode of treatment depends on staging and parametrial infiltration, which is a critical factor in determining treatment planning [10]. Etiological studies have shown that the occurrence of this cancer is a complicated process that includes many factors, and its molecular mechanism has not yet been fully explained. Therefore, there is a need to find an indicator that predicts precancerous changes that lead to cancer [7].

Heat shock proteins (HSPs) and the immune response directed against them are intensively studied by modern oncologists. They are overexpressed in many human malignancies. The heat shock response (HSR) is a basic adaptation of the body to endo- and exogenous stress conditions. It results in the synthesis and increased expression of heat shock proteins [11, 12], which have a protective effect as they neutralise the effects of cellular stress [13]. These proteins protect intracellular proteins from damage leading to their apoptosis [14]. On the other hand, a marked and rapid overexpression of most HSPs in response to cellular damage is found, with cancer cells having been found to over-stimulate the synthesis of heat shock proteins [15]. This may be of fundamental importance in the pathogenesis and progression of cancer - the significant role of heat shock proteins as a therapeutic target in cancer has been demonstrated [16, 17] but, on the other hand, they are also associated with drug resistance in cancer [18].

The endometrium contains many types of heat shock proteins. Among them are HSP27, HSP60, HSP65, HSP70 and HSP90. The intensity of expression of HSP proteins is changeable during the menstrual cycle - there is an inverse correlation between their activity and the 17-ß-estradiol concentration in the human endometrium [19]. Additionally, the association and affinity of HSP27 with the oestrogen receptor in endometrial cancer cells has been demonstrated [20]. Therefore, HSP27 is a protein that may be involved in the pathomechanism of the development of endometrial cancer, especially since it is overexpressed in the neoplastic endometrium [21]. The role of the HSP27 protein in patients is not completely clear. Some authors believe that the presence of increased expression of HSP27 is a favourable prognostic factor, others report that this protein is a marker that significantly decreases the prognosis in terms of treatment efficacy and the recurrence-free period, while still others believe that HSP27 expression is only associated with a good prognosis in endometrial adenocarcinomas, and that the degree of histological differentiation of the endometrial cancer affects HSP27 protein expression - a positive correlation was demonstrated in a well-differentiated cancer [22, 23].

The role of heat shock proteins in cervical cancer has not yet been sufficiently studied and some aspects are still under investigation. The effect of several HSPs in patients with cervical cancer has been analysed in the literature, especially for the HSP70, 60, and 90 groups $[24,25]$. However, the results of studies on heat shock proteins in patients with cervical cancer are heterogeneous.

Although heat shock proteins are promising compounds for the detection, diagnosis and treatment of the analysed cancers, they still require further studies and observations. The aim of this study was to evaluate the concentration of IgG class antibodies for the heat shock proteins HSP27 and HSP60 in the serum of patients with endometrial or cervical cancer, as well as to analyse the variability of the concentrations of the tested antibodies depending on the clinical stage of the cancer (FIGO classification).

\section{MATERIAL AND METHODS}

The study included 59 women with adenocarcinoma of the endometrium and 36 women with cervical cancer. All patients were hospitalised in the Department of Gynaecology, Obstetrics and Gynaecology Oncology, Medical University of Silesia. Patients with coexisting diseases were excluded from the study. The control group consisted of 54 healthy women attending routine gynaecological examinations at the Gynaecological Outpatient Clinic.

For both groups, $5 \mathrm{~mL}$ of blood for analysis was collected from each patient in the morning, on an empty stomach, from the ulnar flexor vein. After collection, the blood was centrifuged, and the serum obtained was stored at $-70^{\circ} \mathrm{C}$ until assayed.

The research protocol was approved by the Bioethics Commission of the Medical University of Silesia, and all patients were informed about the purpose of the study and agreed in writing to participate.

The concentrations of IgG class antibodies against the tested heat shock proteins were determined by an immunoenzymatic assay (ELISA) using commercial assays.

The obtained results are presented using basic parameters of descriptive statistics. The conformity of the distribution of the variables to the normal distribution was checked with the Szapiro-Wilk test. Nonparametric Kolmogorov-Smirnov and Mann-Whitney $U$ tests were used for comparisons between the groups. The Kruskal-Wallis rank-sum ANOVA test was used to examine variability within 
the endometrial cancer group. The correlation between parameters was examined using Spearman's rank correlation test. A p value less than 0.05 was considered statistically significant. The Statistica computer program (StatSoft Inc.) was used for calculations.

\section{RESULTS}

The average age of the patients with endometrial or cervical cancer and the healthy women was similar and not statistically different $(p>0.05)$. Similarly, there was no significant difference between the ages of patients in the different stages of cancer (Tab. 1). Table 1 presents the structure of the group of women with endometrial or cervical cancer depending to the clinical stage of the neoplastic process according to FIGO classification. In the case of endometrial cancer, both FIGO stages I and II were diagnosed in 21 patients (35.6\% each), while stage IV was the least frequently found (5 patients, i.e., $8.5 \%$ of cases). A slightly different tumour progression was observed in the patients with cervical cancer. In this group, the most frequent tumour grade was II - almost half of the patients ( $47.2 \%$ of cases).

After the assays were performed and the results obtained, the concentrations of the tested antibodies in the serum of women with endometrial adenocarcinoma or cervical cancer were compared with the concentrations of these antibodies in the serum of the women in the control group. The results of these comparisons are presented in Table 2 .

In both endometrial and cervical cancer patients, the concentrations (medians) of IgG antibodies to HSP27 ( $p<0.01$ and $p=0.026$ for the Kolmogorov-Smirnov and Mann-Whitney
U tests, respectively, for endometrial cancer and $p=0.022$ and $p=0.041$ for the Kolmogorov-Smirnov and Mann-Whitney $U$ tests, respectively, for cervical cancer) were significantly higher than in the control group of healthy women. However, for both cancers, the IgG anti-HSP60 antibody levels were comparable in both groups (Tab. 2).

Table 3 presents the concentrations of the tested antibodies (medians) depending on the clinical stage of the neoplastic process assessed according to the FIGO classification.

In the patients with endometrial cancer, anti-HSP60 antibody concentrations in those women with FIGO I and FIGO II are significantly lower than in those with FIGO IV $(p=0.019$ and $p=0.024$, respectively). There were no differences between the anti-HSP27 antibody levels in this group of patients. The results of the cervical cancer patients are slightly different. In this group, we observed a decrease in the concentrations of the tested antibodies depending on the tumour stage. Significantly higher concentrations of anti-HSP27 were observed in those women with FIGO I and FIGO II than in those with FIGO IV ( $p=0.0001$ and $p=0.003$, respectively) and higher concentrations of anti-HSP60 in those women with FIGO I and FIGO III than in those with FIGO IV ( $p=0.023$ and $p=0.0011$, respectively). Due to the inverse trend of the behaviour of the concentrations of the tested antibodies in both diseases, they were not compared with each other.

In patients with endometrial cancer at clinical stage I according to FIGO, the concentrations of the tested antibodies were analysed in relation to the depth of tumour infiltration. The results of this analysis are presented in Table 4. It turned

\begin{tabular}{|c|c|c|c|c|}
\hline & \multicolumn{4}{|c|}{ Clinical stage of endometrial cancer according to FIGO } \\
\hline & I & II & III & IV \\
\hline Group size (n) & 21 & 21 & 12 & 5 \\
\hline Percentage share [\%] & 35.6 & 35.6 & 20.3 & 8.5 \\
\hline \multirow[t]{3}{*}{ Average age [years] $\pm S D$} & $61.2 \pm 11.6$ & $61.6 \pm 8.0$ & $64.7 \pm 7.2$ & $55.4 \pm 9.2$ \\
\hline & \multicolumn{4}{|c|}{ Clinical stage of cervical cancer according to FIGO } \\
\hline & I & II & III & IV \\
\hline Group size (n) & 13 & 17 & 5 & 1 \\
\hline Percentage share [\%] & 36.1 & 47.2 & 13.9 & 2.8 \\
\hline Average age [years] $\pm S D$ & $51.4 \pm 6.6$ & $53.2 \pm 7.9$ & $59.9 \pm 11.8$ & 58 \\
\hline
\end{tabular}

SD - standard deviation

Table 2. Concentrations of $\operatorname{lgG}$ antibodies against tested heat shock proteins in endometrial cancer patients and cervical cancer patients and controls

\begin{tabular}{|l|l|l|l|}
\hline Tested parameter median, range $[\mathrm{AU} / \mathrm{mL}]$ & Endometrial cancer & Cervical cancer & Control group \\
\hline anti-HSP27 & $74.3^{*}(9.6-506.3)$ & $58.0^{*}(6.9-914.9)$ & $43.2(4.8-240.2)$ \\
\hline anti-HSP60 & $50.2(2.7-853.7)$ & $43.2(2.0-494.8)$ & $48.6(9.4-305.7)$ \\
\hline
\end{tabular}

${ }^{*} p<0.05$ compared to a control group 


\begin{tabular}{|l|l|l|l|l|l|}
\hline \multicolumn{5}{|l|}{ Table 3. Concentrations of the tested antibodies according to the clinical stage of the neoplastic process according to FIGO } \\
\hline \multirow{2}{*}{ Tested parameter - median [AU/mL] } & \multicolumn{4}{|l|}{ Clinical stage of endometrial cancer according to FIGO classification } \\
\cline { 2 - 5 } & I & II & III & IV \\
\hline anti-HSP27 & 41.2 & 63.2 & 76.9 & 113.4 \\
\hline anti-HSP60 & $35.1^{*}$ & $43.8^{*}$ & 52.7 & 158.5 \\
\hline \multirow{2}{*}{ Tested parameter - median [AU/mL] } & Clinical stage of cervical cancer according to FIGO classification & III \\
\hline anti-HSP27 & I & II & $71.9^{*}$ & 56.1 & 11.1 \\
\hline anti-HSP60 & $134.4^{*}$ & 9.2 & $56.9^{*}$ & 8.5 \\
\hline
\end{tabular}

${ }^{*} \mathrm{p}<0.05$ compared to FIGO IV group

Table 4. Levels of tested antibodies according to the depth of endometrial infiltration within FIGO group I in patients with endometrial cancer

\begin{tabular}{|l|l|l|}
\hline $\begin{array}{l}\text { Tested parameter } \\
\text { median, range } \\
\text { [AU/mL] }\end{array}$ & \multicolumn{2}{|l|}{ Clinical stage according to FIGO } \\
\cline { 2 - 3 } anti-HSP27 & IA & IB \\
\hline anti-HSP60 & $33(11.7-132.8)$ & $46^{*}(19.3-203.8)$ \\
\hline
\end{tabular}

${ }^{*} \mathrm{p}<0.05$ compared to FIGO IA group

out that in cases of neoplastic infiltration of less than $50 \%$ of the endometrium (FIGO stage IA), the concentration of antibodies in the lgG group is lower than in the case of clinical stage IB, where half or more of the endometrial thickness is affected by the neoplastic process. The mentioned relationship concerns both anti-HSP27 and anti-HSP60 antibodies.

An assessment of the possible correlations of the concentrations of the tested antibodies in ill and healthy women with their age and an assessment of the possible correlations between the concentrations of antibodies against particular HSPs in the examined groups were also performed. The concentrations of anti-HSP27 and anti-HSP60 antibodies did not correlate with the age of the women in any of the examined groups. The concentrations of the tested antibodies correlated positively with each other, both in those women with cancer and in the healthy group. The strongest correlations were found in the group of patients with endometrial cancer. The results are shown in Table 5.

\section{DISCUSSION}

In our study of endometrial cancer, the largest group was for patients with an earlier tumour stage according to FIGO (stages I and II). Similar results were presented by Hou et al. [26], with $73.9 \%$ of patients at stages I and II, and the rest of the group studied in his report (stages III and IV) accounting for $26.1 \%$, as well as by Helpman et al. [27], who in their large study of more than 20,000 patients with endometrial cancer describe that $73 \%$ of tumours were confined to the uterus, so the stage was I or II. A slightly
Table 5. Evaluation of the correlation between the concentrations of studied antibodies in the group of endometrial cancer patients and healthy women

\begin{tabular}{|c|c|c|}
\hline & anti-HSP27 & anti-HSP60 \\
\hline \multicolumn{3}{|c|}{ Endometrial cancer } \\
\hline anti-HSP27 & - & $\begin{array}{l}R=0.558 \\
p=0.000005\end{array}$ \\
\hline \multicolumn{3}{|c|}{ Cervical cancer } \\
\hline anti-HSP27 & - & $\begin{array}{l}R=0.328 \\
p=0.0004\end{array}$ \\
\hline \multicolumn{3}{|c|}{ Healthy women } \\
\hline anti-HSP27 & - & $\begin{array}{l}R=0.499 \\
p=0.00012\end{array}$ \\
\hline
\end{tabular}

$\mathrm{p}$ - level and Spearmann's; R - correlation coefficient

different cross-section of tumour progression was obtained by Van Walree et al. [28], who report that FIGO stage I patients comprise as many as $88 \%$ of the study participants, while stage II patients comprise only $3 \%$. This difference is probably due to the broad exclusion criteria used in the Van Walree et al., study; some patients with progressive disease were excluded from participation. In our study, we also obtained a similar cross section of cancer stage for patients with endometrial cancer, as have other authors [8].

The primary objective of this study was to evaluate the concentrations of IgG class antibodies against selected heat shock proteins in the serum of women with endometrial or cervical cancer.

The study in endometrial cancer patients focused on type 1 endometrial cancer due to it affecting $80 \%$ of patients. In our study, IgG anti-HSP27 antibody levels were higher in the study group compared to the healthy women. The results are consistent with the reports of other authors, as overexpression of anti-HSP27 antibodies was detected in cases of endometrial cancer [29]. Heat shock proteins protect against the effect of exogenous stress, which translates into their higher concentration in endometrial cancer patients (and other cancers) [30]. The results of the concentrations of these proteins in women with epithelial 
endometrial cancer, different to ours, were presented by Boeddeker et al. [31]. In their study, before apoptosis induction, HSP60 concentrations were lower in women with endometrial cancer in comparison to healthy controls, and HSP27 concentrations did not differ between the groups. After the induction of apoptosis, the concentrations of the tested antibodies were lower in women with endometrial cancer. The results of Boeddeker et al. can be explained by the fact that the HSP27 protein is considered an inhibitory factor for apoptosis, which has also been mentioned by other authors [30, 32, 33].

In their studies, Ramezani along with Aghakhani et al. [30], showed higher anti-HSP27 antibody levels in women exposed to HPV than in the control group. This is consistent with our results and demonstrates that HSP27 is a biomarker for HPV infection as well as a therapeutic target for cancer [30, 34].

In our study, we obtained a correlation of a clear increase in antibody concentrations with the FIGO stage of endometrial cancer. A particularly pronounced increase was noted in the case of anti-HSP60. Similar conclusions have been reached by other studies. It appears that extracellular HSP proteins and HSP-rich extracellular vesicles can promote cancer progression. Moreover, they enhance migration, invasion, heterogeneity, metastasis, and drug resistance in cancer cells, but most importantly they promote tumour angiogenesis $[35,36]$. The cited facts clearly explain the increase in the concentrations of HSP proteins and, consequently, the antibodies tested with the stage of this cancer. In the case of cervical cancer, we obtained results suggesting a decrease in the concentration of anti-HSP27 antibodies with tumour progression. This is probably due to the inhibitory effect of lambertian acid on HSP27 phosphorylation and, consequently, to the impaired binding of HSP27 to the factors promoting its synthesis, as described by Lee et al. [37]. It should also be remembered that cervical cancer develops slowly - therefore we found the highest concentration of anti-HSP27 antibodies in FIGO stage I, as in the case of a slow-growing cancer an enhanced autoimmune response may be expected in the early stages of its development. We believe that the enhanced nature of the immune response against HSP27 in patients with cervical cancer may indicate an increased expression of this protein, especially in the early stages of the disease and suggests its involvement in the oncogenesis process of this cancer.

We did not get a clear answer why the behaviour of anti-HSP60 antibodies in women with cervical cancer depends on the stage - the highest levels were found in the FIGO III group, while the lowest levels were found in the FIGO II and IV groups. Perhaps such inconsistent results are due to the limited number of patients studied, but in the literature on the subject, similar behaviour of these chemical compounds is pointed out [38]. We believe that the analysis of heat shock protein concentrations and the antibodies directed against them in women with cervical cancer requires further study.

We also noted an increase in the concentrations of the tested antibodies with increasing depth of endometrial infiltration in women with endometrial cancer. This observation seems to be important because the depth of myometrial infiltration may determine the qualification for the extension of surgery. The removal of the ovaries may be abandoned in women under the age of 45 with radiological or FIGO IA stage endometrial cancer $[39,40]$.

We were able to prove the existence of positive correlations between all the tested antibodies for those patients with endometrial cancer. This is a consequence of the promotion of oncogenesis by multiple types of HSP proteins in cancer cells and the local tumour environment in the uterus $[41,42]$. Heat shock factor 1 (HSF1) plays a major role here, driving the transcription of heat shock proteins and triggering their massive synthesis, enhancing oncogenesis [43]. It is also important in cervical cancer patients [44], as confirmed by the correlations found in this group as well. It has been shown that in patients with cervical cancer, an increase in HSF1 activity is associated with resistance to chemotherapy drugs or a bad prognosis [45]. In our study, we were able to obtain an increase in only one of the antibodies tested in FIGO stage III, and thus a likely increase in HSF1 in this very group.

A cardinal challenge in the diagnosis of genital cancers is the discovery of better markers to assist in the identification of patients with early-stage cancer. Few biochemical prognostic markers are available for the two cancers analysed and none has entered clinical practice permanently. To resolve this issue, the biological processes underlying the progression of both cancers need to be better understood. The presence of heat shock proteins and anti-HSP antibodies in the serum of cancer patients is still an emergent area of research. Although autoantibodies to certain HSPs already appear to have relevance as tumour markers in osteosarcoma, ovarian cancer, and others, we are now in need of more studies to conclude clearly on this important subject.

\section{CONCLUSIONS}

The authors conclude that anti-HSP27 and anti-HSP60 antibodies may therefore be biomarkers of carcinogenesis for endometrial and cervical cancer. The concentration of antibodies against these proteins may indirectly indicate the cancer stage. The existence of a clear positive correlation between the levels of the tested antibodies may indicate a similar nature of the participation of HSP27 and HSP60 in the pathogenesis of these tumours. We believe that the analysis of heat shock protein concentrations and the antibodies directed against them in women with endometrial or cervical cancer requires further studies. 


\section{Conflict of interest}

The authors state that there are no conflicts of interest to disclose.

\section{REFERENCES}

1. Chen JLY, Huang YS, Huang CY, et al. Impact of adjuvant radiotherapy on the survival of women with optimally resected stage III endometrial cancer in the era of modern radiotherapy: a retrospective study. Radiat Oncol. 2020; 15(1): 72, doi: 10.1186/s13014-020-01523-5, indexed in Pubmed: 32252781.

2. Muzykiewicz KP, Iwanska $E$, Janeczek $M$, et al. The analysis of the prognostic value of the neutrophil/ lymphocyte ratio and the platelet/lymphocyte ratio among advanced endometrial cancer patients. Ginekol Pol. 2021; 92(1): 16-23, doi: 10.5603/GP.a2020.0164, indexed in Pubmed: 33448001.

3. Anton $\mathrm{C}$, Kleine RT, Mayerhoff $\mathrm{E}$, et al. Ten years of experience with endometrial cancer treatment in a single Brazilian institution: Patient characteristics and outcomes. PLoS One. 2020; 15(3): e0229543, doi: 10.1371/journal.pone.0229543, indexed in Pubmed: 32134921.

4. Amant F, Moerman P, Neven P, et al. Endometrial cancer. The Lancet. 2005; 366(9484): 491-505, doi: 10.1016/s0140-6736(05)67063-8.

5. Reijnen C, van der Putten LJM, Bulten J, et al. Mutational analysis of cervical cytology improves diagnosis of endometrial cancer: A prospective multicentre cohort study. Int J Cancer. 2020; 146(9): 2628-2635, doi: 10.1002/ijc.32686, indexed in Pubmed: 31523803.

6. Zhang J, Si J, Gan Lu, et al. Inhibition of Wnt signalling pathway by XAV939 enhances radiosensitivity in human cervical cancer HeLa cells. Artif Cells Nanomed Biotechnol. 2020; 48(1): 479-487, doi: 10.1080/21691401.2020.1716779, indexed in Pubmed: 31975621.

7. Chen $\mathrm{H}$, Wang $\mathrm{Xi}$, Jia $\mathrm{H}$, et al. Bioinformatics analysis of key genes and pathways of cervical cancer. Onco Targets Ther. 2020; 13: 13275-13283, doi: 10.2147/OTT.S281533, indexed in Pubmed: 33402836.

8. Qi YX, Liu K, Yin J, et al. Evaluation of short- and long-term efficacy of chemoradiotherapy for advanced cervical cancer using HSP70 protein combined with multimodal MRI. J Cell Biochem. 2018; 119(4): 3017-3029, doi: 10.1002/jcb.26424, indexed in Pubmed: 29023986.

9. de Freitas AC, Gurgel A, Chagas B, et al. Susceptibility to cervical cancer: An overview. Gynecologic Oncology. 2012; 126(2): 304-311, doi: 10.1016/j.ygyno.2012.03.047

10. Tomalczyk A, Tomasik B, Moszynska-Zielinska M, et al. The assessment of spectral Doppler parameters in uterine arteries of patients with locally advanced squamous cell cervical cancer. Ginekol Pol. 2019; 90(11): 622-627, doi: 10.5603/GP.2019.0101, indexed in Pubmed: 31802461.

11. Singh IS, Hasday JD, Hasday JD, et al. Fever and the heat shock response: distinct, partially overlapping processes. Cell Stress Chaperones. 2000; 5(5): 471-480, doi: 10.1379/1466-1268(2000)005<0471:fathsr>2.0.co;2, indexed in Pubmed: 11189454.

12. Kim HJ, Hwang NaR, Lee KJ. Heat shock responses for understanding diseases of protein denaturation. Mol Cells. 2007; 23(2): 123-131, indexed in Pubmed: 17464187.

13. Horowitz $M$, Robinson SDM. Heat shock proteins and the heat shock response during hyperthermia and its modulation by altered physiological conditions. Prog Brain Res. 2007; 162: 433-446, doi: 10.1016/S00796123(06)62021-9, indexed in Pubmed: 17645931.

14. Dubrez L, Causse S, Borges Bonan N, et al. Heat-shock proteins: chaperoning DNA repair. Oncogene. 2020; 39(3): 516-529, doi: 10.1038/s41388019-1016-y, indexed in Pubmed: 31541194.

15. Stangl S, Tontcheva N, Sievert W, et al. Heat shock protein 70 and tumor-infiltrating NK cells as prognostic indicators for patients with squamous cell carcinoma of the head and neck after radiochemotherapy: A multicentre retrospective study of the German Cancer Consortium Radiation Oncology Group (DKTK-ROG). Int J Cancer. 2018; 142(9): 1911-1925, doi: 10.1002/ijc.31213, indexed in Pubmed: 29235112.

16. Brünnert $D$, Langer $C$, Zimmermann $L$, et al. The heat shock protein 70 inhibitor VER155008 suppresses the expression of HSP27, HOP and HSP90 $\beta$ and the androgen receptor, induces apoptosis, and attenuates prostate cancer cell growth. J Cell Biochem. 2020; 121(1): 407-417, doi: 10.1002/jcb.29195, indexed in Pubmed: 31222811.

17. Rottach AM, Ahrend H, Martin B, et al. Cabazitaxel inhibits prostate cancer cell growth by inhibition of androgen receptor and heat shock protein expression. World J Urol. 2019; 37(10): 2137-2145, doi: 10.1007/s00345-018-2615-x, indexed in Pubmed: 30603780.
18. Hoter A, Naim HY. Heat shock proteins and ovarian cancer: important roles and therapeutic opportunities. Cancers (Basel). 2019; 11(9), doi: 10.3390/cancers11091389, indexed in Pubmed: 31540420.

19. Guzel E, Basar M, Ocak N, et al. Bidirectional interaction between unfolded-protein-response key protein HSPA5 and estrogen signaling in human endometrium. Biol Reprod. 2011;85(1): 121-127, doi: 10.1095/biolreprod.110.089532, indexed in Pubmed: 21389343.

20. Ciocca DR, Calderwood SK. Heat shock proteins in cancer: diagnostic prognostic, predictive, and treatment implications. Cell Stress Chaperones. 2005; 10(2): 86-103, doi: 10.1379/csc-99r.1, indexed in Pubmed: 16038406.

21. Wataba K, Saito T, Fukunaka K, et al. Over-expression of heat shock proteins in carcinogenic endometrium. Int J Cancer. 2001; 91(4): 448-456, doi: 10.1002/1097-0215(200002)9999:9999<::aid-ijc1077>3.0.co;2-f, indexed in Pubmed: 11251965.

22. Geisler JP, Tammela JE, Manahan KJ, et al. HSP27 in patients with ovarian carcinoma: still an independent prognostic indicator at 60 months follow-up. Eur J Gynaecol Oncol. 2004; 25(2): 165-168, indexed in Pubmed: 15032273.

23. Heidari F, Rabizadeh S, Sadat Salehi S, et al. Serum HSP70 level in patients with endometrial cancer with and without diabetes. Gynecol Endocrinol. 2020; 36(4): 351-355, doi: 10.1080/09513590.2019.1648415, indexed in Pubmed: 31392909.

24. Jakubowicz-Gil J, Paduch R, Skalicka-Woźniak K, et al. Hsps responsible for apoptosis induction failure in cervical cancer cells upon osthole and tamoxifen treatment. Postępy Higieny i Medycyny Doświadczalnej. 2019; 73: 563-571, doi: 10.5604/01.3001.0013.5447.

25. Han S, Cheng Z, Zhao X, et al. Diagnostic value of heat shock protein 90 and squamous cell carcinoma antigen in detection of cervical cancer. JInt Med Res. 2019; 47(11):5518-5525, doi: 10.1177/0300060519865634, indexed in Pubmed: 31426690.

26. Hou Y, Li T, Gan W, et al. Prognostic significance of mutant-allele tumor heterogeneity in uterine corpus endometrial carcinoma. Ann Transl Med. 2020; 8(6): 339, doi: 10.21037/atm.2020.02.136, indexed in Pubmed: 32355783.

27. Helpman L, Perri T, Lavee N, et al. Impact of adjuvant treatment on outcome in high-risk early-stage endometrial cancer: a retrospective three-center study. Int J Gynecol Cancer. 2019; 29(1): 133-139, doi: 10.1136/ijgc-2018-000030, indexed in Pubmed: 30640695.

28. van Walree IC, Hamaker ME, de Rooij BH, et al. Do age and comorbidity impair recovery during two years after treatment for endometrial cancer? J Geriatr Oncol. 2020; 11(7): 1078-1086, doi: 10.1016/j.jgo.2020.02.012, indexed in Pubmed: 32169547.

29. Ioachin E. Immunohistochemical tumour markers in endometrial carcinoma. Eur J Gynaecol Oncol. 2005; 26(4): 363-371, indexed in Pubmed: 16122180.

30. Ramezani A, Aghakhani A, Soleymani S, et al. Significance of serum antibodies against HPVE7, Hsp27, Hsp20 and Hp91 in Iranian HPV-exposed women. BMC Infect Dis. 2019; 19(1): 142, doi: 10.1186/s12879-019-37802, indexed in Pubmed: 30755156.

31. Boeddeker SJ, Baston-Buest DM, Altergot-Ahmad O, et al. Syndecan-1 knockdown in endometrial epithelial cells alters their apoptotic protein profile and enhances the inducibility of apoptosis. Mol Hum Reprod. 2014; 20(6): 567-578, doi: 10.1093/molehr/gau009, indexed in Pubmed: 24478463.

32. Neuer A, Spandorfer SD, Giraldo P, et al. The role of heat shock proteins in reproduction. Hum Reprod Update. 2000; 6(2): 149-159, doi: 10.1093/humupd/6.2.149, indexed in Pubmed: 10782573.

33. Garrido $C$, Brunet $M$, Didelot $C$, et al. Heat shock proteins 27 and 70 : anti-apoptotic proteins with tumorigenic properties. Cell Cycle. 2006; 5(22): 2592-2601, doi: 10.4161/cc.5.22.3448, indexed in Pubmed: 17106261.

34. Vidyasagar A, Wilson NA, Djamali A. Heat shock protein 27 (HSP27): biomarker of disease and therapeutic target. Fibrogenesis Tissue Repair. 2012; 5(1): 7, doi: 10.1186/1755-1536-5-7, indexed in Pubmed: 22564335.

35. Najafi M, Goradel NH, Farhood B, et al. Tumor microenvironment: Interactions and therapy. J Cell Physiol. 2019; 234(5): 5700-5721, doi: 10.1002/jcp.27425, indexed in Pubmed: 30378106.

36. Nolan KD, Franco OE, Hance MW, et al. Tumor-secreted Hsp90 subverts polycomb function to drive prostate tumor growth and invasion. J Biol Chem. 2015; 290(13): 8271-8282, doi: 10.1074/jbc.M115.637496, indexed in Pubmed: 25670862.

37. Lee JC, Sim DY, Lee HJ, et al. MicroRNA216b mediated downregulation of HSP27/STAT3/AKT signaling is critically involved in lambertianic acid induced apoptosis in human cervical cancers. Phytother Res. 2021; 35(2): 898-907, doi: 10.1002/ptr.6842, indexed in Pubmed: 32822082. 
38. Seigneuric R, Mjahed H, Gobbo J, et al. Heat shock proteins as danger signals for cancer detection. Front Oncol. 2011; 1: 37, doi: 10.3389/fonc.2011.00037, indexed in Pubmed: 22649762.

39. Sznurkowski J, Knapp P, Bodnar L, et al. Recommendations of the Polish Gynecological Oncology Society for the diagnosis andtreatment of endometrial cancer. Current Gynecologic Oncology. 2017; 15(1):34-44, doi: $10.15557 / \mathrm{cgo} .2017 .0003$.

40. Colombo N, Creutzberg C, Amant F, et al. ESMO-ESGO-ESTRO Endometrial Consensus Conference Working Group. ESMO-ESGO-ESTRO Consensus Conference on Endometrial Cancer: Diagnosis, Treatment and Follow-up. Int J Gynecol Cancer. 2016; 26(1): 2-30, doi: 10.1097/IGC.0000000000000609, indexed in Pubmed: 26645990.

41. Ono K, Eguchi T, Sogawa C, et al. HSP-enriched properties of extracellular vesicles involve survival of metastatic oral cancer cells. J Cell Biochem. 2018; 119(9): 7350-7362, doi: 10.1002/jcb.27039, indexed in Pubmed: 29768689.
42. Fujiwara T, Eguchi T, Sogawa C, et al. Carcinogenic epithelial-mesenchymal transition initiated by oral cancer exosomes is inhibited by anti-EGFR antibody cetuximab. Oral Oncol. 2018; 86: 251-257, doi: 10.1016/j. oraloncology.2018.09.030, indexed in Pubmed: 30409309.

43. Engerud $\mathrm{H}$, Tangen IL, Berg A, et al. High level of HSF1 associates with aggressive endometrial carcinoma and suggests potential for HSP90 inhibitors. Br J Cancer. 2014; 111(1): 78-84, doi: 10.1038/bjc.2014.262, indexed in Pubmed: 24853175.

44. Yu S, Cheng M, Hou L, et al. HSF1 expression in cervical carcinoma and its correlation with clinical pathological characteristics and prognosis. J Third Mil Med Univ. 2017; 36: 560-563.

45. Zhang L, Hu Z, Zhang Y, et al. Proteomics analysis of proteins interacting with heat shock factor 1 in squamous cell carcinoma of the cervix. Oncol Lett. 2019; 18(3): 2568-2575, doi: 10.3892/ol.2019.10539, indexed in Pubmed: 31402952 